\title{
Preparation and Characterization of Lidocaine Double Layer Buccal Tablet Using Mucoadhesive Carbopol ${ }^{\circledR}$ Polymers
}

\author{
Nahid Sharmin, Md.Elias-Al-Mamun, Md.Saiful Islam and Reza-ul J alil \\ Department of Pharmaceutical Technology, Faculty of Pharmacy, University of Dhaka, \\ Dhaka-1000, Bangladesh
}

\begin{abstract}
The present study was intended to develop a new oral mucosal dosage form with a view of solving problems of the topical administration of Lidocaine, a local anesthetic drug, in the oral cavity. The drug was formulated as sustained release mucoadhesive bilayered tablets using mucoadhesive polymers. The first layer, consisting of a drug impermeable backing, was prepared by compression of Kollidon SR, Mg-stearate, Aerosil and talc blend in a hydrolic press at 5 ton pressure for 5 seconds. The second layer, responsible for buccal drug delivery and tablet retention on the mucosa was obtained by compression of a mixture of drug, mucoadhesive polymer and cellulose derivative for $1 \mathrm{~min}$ at 5 ton pressure. The tablets were evaluated in terms of swelling and water uptake and in vivo mucoadhesive performance was performed with nonmedicated tablets. In vitro drug release studies were also carried out containing fixed amount of Lidocaine (50mg), different concentrations of mucoadhesive polymer (Carbopol 934P) and hydroxypropyl methyl cellulose (HPMC). Drug release was only $30.9 \%$ in 6 hours when Carbopol 934P was used alone and at a higher concentration (240mg). Release was faster when lower concentration of polymer was used. When used in combination with HPMC, drug release was slower. In vivo mucoadhesive performance with nonmedicated tablets showed very good adherence to buccal mucosa for 6 hours and no signs of irritation was reported. Moreover, it adheres well to the gum, is not washed away by salivary fluid and is simple to apply, which indicates a better patient compliance.
\end{abstract}

Key words: Bilayer tablets, Mucoadhesion, Carbopol polymers, Lidocaine, HPMC etc.

\section{INTRODUCTION}

Adhesion is defined by the physicist as the molecular force of attraction in the area of contact between unlike bodies that acts to hold them together ${ }^{1}$ and Bioadhesion is defined as the state in which two bodies, one or both of the adherends, are of a biological nature and are held together for extended periods of time by interfacial forces which may range from valence forces to mechanical interactions, or combination of chemical and physical interactions. A bioadhesive can therefore be defined as a substance which has the ability to interact with

Correspondence to: Nahid Sharmin

E-mail: sharminnahid@yahoo.com

Dhaka Univ. J. Pharm. Sci. 10(1): 29-34, 2011 (June) biological materials and is capable of being retained on the biological substrate for a period of time. ${ }^{2}$ When the polymeric system interacts with mucus layer only, it is referred to as mucoadhesion. ${ }^{3}$ The concept of mucoadhesion was introduced into controlled drug delivery in the early 1980's. ${ }^{4}$

Bioadhesive systems have been used for many years in dentistry and orthopaedics as well as in ophthalmology and for surgical applications. However, recently there has been also a significant interest in the use of bioadhesives in other areas, such as soft tissue-based artificial replacements and controlled release systems for local release of bioactive agents. Such applications include systems for release of drugs in the buccal or nasal cavity, for 
intestinal or rectal administration, and even for urinary bladder applications. ${ }^{5}$ Although the buccal mucosa is less permeable than the sublingual mucosa and it does not yield a rapid onset of action as sublingual delivery, mucosa of the buccal area has a smooth and relatively immobile surface, which is suitable for placement of a retentive system. ${ }^{6}$ Buccal delivery can give rapid absorption, avoids first-pass metabolism of the drug, avoids degradation in the stomach (from acid or from gastric enzymes) and subjects the drug to a milder enzyme milieu. Drug delivery can be efficient, since the mucosal surface is washed with 0.5 to 2 liters/day of saliva, and the absorption area can be a reasonable fraction of the maximum available. However, drug delivery can also be precisely localized if a device is held in position with a mucoadhesive and is placed away from the salivary glands. ${ }^{7}$

In this study Carbopol polymers have been used as mucoadhesives. Carbopol polymers are high molecular weight, crosslinked, acrylic acid-based polymers. All of the carbopol polymers have the same acrylic acid backbone. The main differences are related to the presence of a comonomer and the crosslink density. With very minor adjustments in the crosslink density and comonomer level, a large number of polymers have been engineered to provide specific application properties without substantially changing the gross molecular structure. ${ }^{8}$ Carbopol 934P is cross-linked with allyl sucrose and is polymerized in solvent benzene. Carbopol 971P, 974P are cross-linked with allyl penta erythritol and polymerized in ethyl acetate. Carbopol $971 \mathrm{P}$ has lower level of crosslinking density than Carbopol 934P and Carbopol 974P. ${ }^{9}$ The purpose of this study was to design a buccal drug delivery system using Lidocaine and a buccal adhesive and to evaluate the feasibility of a sustained transbuccal drug delivery system. Inflammatory processes cause most oral cavity diseases. ${ }^{10}$ This problem is managed with the topical administration of Lidocaine, an amide local anesthetic.

\section{MATERIALS AND METHODS}

Materials. Kollidon SR was obtained from BASF (Germany) and HPMC (15 cps) was supplied by Samsung (Korea)). Carbopol 934P, 974P and Carbopol 971P were obtained from Noveon (USA). HPC was obtained from DOW (USA). Iron Oxide Red was purchased from BASF (Germany). All other materials were of reagent grade.

Bilayered tablet preparation. The powder blends for tablets were prepared by triturating in a mortar and pestle, and bilayer tablets were prepared in 2 stages using a 13-mm-diameter die on an infrared hydraulic press (Perkin-Elmer, Cambridge, UK) with a compression force of $5 \times 10^{3} \mathrm{~kg}$ for a total time of 65 seconds. Initially, $162 \mathrm{mg}$ of the nonmucoadhesive polymer blend (Table 1) was compressed for 5 seconds, known as first tableting time to obtain the drug impermeable layer. Iron Oxide Red was added to mark the layer. Following first compression, the punch was lifted, $300 \mathrm{mg}$ of the second-layer blend (Table 1) was added, and a second compression $\left(5 \times 10^{3} \mathrm{~kg}\right)$ was performed for 60 seconds referred to as second tableting time to obtain the bilayered tablet.

In vitro water uptake studies. 3.5 inch diameter PVC pipe was cut into small pieces of about $1 / 4$ inch height. Stainless steel mesh were cut according to the diameter of the pipe. These meshes were attached at the bottom of the pieces of the pipe by glue. The device was then weighed (W1). The tablets were kept upon the device keeping the colored part up-side (Figure 1) and reweighed (W2). Difference between W2 and W1 indicates weight of tablets. The tablets along with the devices were then immersed in three petridishes containing distilled water, $0.1 \mathrm{~N} \mathrm{HCl}$ and pH 6.8 phosphate buffer. Eevery hour, the assembly were raised out of the petridish, the water droplets that adhered to the surface of the assembly was wiped off and reweighed (W3). Difference between W3 and W2 indicates increase in weight of the tablets. Percentage of water uptake was calculated using the formula :

Amount of water uptake $=(\mathrm{W} 3-\mathrm{W} 2) \times 100 /(\mathrm{W} 2-\mathrm{W} 1)$ 
In vitro swelling studies. Initially, the radial diameter of the tablets was $13 \mathrm{~mm}$ (D1). To observe the radial swelling of the tablets, the bilayered tablets were immersed in distilled water, $0.1 \mathrm{~N} \mathrm{HCl}$, and 6.8 $\mathrm{pH}$ phosphate buffer for 6 hours and images of the tablets were taken at every hour interval with a digital camera (SONY, 8.1 megapixel, Japan). Using Microsoft Visio (version 2007) software the diameter of the tablets was determined for each hour (D2). From these data percentage of swelling of the tablets was calculated in percentage using the following formula :

$\%$ Swelling $=(\mathrm{D} 2-\mathrm{D} 1) \times 100 / \mathrm{D} 1$

In vivo mucoadhesive performance of tablets without drug. To observe the in vivo mucoadhesive performance, bilayered tablets without drug were prepared. The formulations were the same as described in table 1 except that Lidocaine was not added in the formulations. In vivo mucoadhesive studies were performed by applying bilayered tablets on 5 healthy volunteer gums to assess the residence time and the possible production of irritation or pain. The non-colored part of the tablet i.e. the second layer of the bilayered tablet containing mucoadhesive polymer was attached to the gum of the volunteers (Figure 2). All subjects were healthy, not taking any medications, and had no history of drug related allergies. Each tablet was attached to the gum by applying a light force with a fingertip for 20 seconds. Tablet behavior and mucoadhesion time were monitored.

\section{In vitro drug release study}

Drug-loaded tablet preparation. Two series of bilayered drug loaded tablets were prepared containing $50 \mathrm{mg}$ of Lidocaine. Formulation F1, F2 and F3 contained Carbopol 934P only (Table 2). Formulation F4, F5 and F6 were prepared with Carbopol 934P and HPMC (Table 2) .

In vitro release study. In vitro drug release study was performed in $100 \mathrm{ml}$ vials. $50 \mathrm{ml}$ of $\mathrm{pH} 6.8$ phosphate buffer was taken in the vials and used as dissolution media. Dissolution vessels of a dissolution testing machine were filled with water and temperature was fixed at $37 \pm 5^{\circ} \mathrm{C}$. The vials were held with a test tube holder and kept within the dissolution vessels, so that temperature of the vials can be maintained at $37 \pm 5^{\circ} \mathrm{C}$. Each tablet with the drug permeable backing at the bottom (the noncolored part) was placed into the vials. The dissolution process was carried out for 6 hours. $5 \mathrm{ml}$ of dissolution sample from each batch was withdrawn at every 10 minutes for 1 hour and then every 30 minutes for 5 hours. In each case $5 \mathrm{ml}$ of dissolution sample was compensated by another fresh $5 \mathrm{ml}$ phosphate buffer. The dissolution samples were then analyzed spectrophotometrically with UV-VIS spectrophotometer (UV mini-1240, SHIMADZU CORP., Kyoto, Japan) at $263 \mathrm{~nm}$. The dissolution study was done 2 times for each batch.

\section{RESULTS AND DISCUSSION}

In vitro water uptake Studies. Initially the weight of the tablets was $200 \mathrm{mg}$. All tablets started hydration very quickly. After 6 hours, \% hydration of the tablets containing Carbopol 934P was $469 \%$ in distilled water, whereas in $0.1 \mathrm{~N} \mathrm{HCl}$ and in $\mathrm{pH} 6.8$ phosphate buffer they were $401 \%$ and $461 \%$ respectively. For tablets containing Carbopol 974P and 971P after 6 hours, \% hydration were $458 \%$ and $476 \%$ respectively in distilled water, $383 \%$ and $433 \%$ in $0.1 \mathrm{~N} \mathrm{HCl}$ and $460 \%$ and $597 \%$ in $\mathrm{pH} 6.8$ phosphate buffer respectively. From these data it is evident that, Carbopol polymers can absorb gastric fluid, water and GIF many times of their weight which is very important to be considered because water penetration is responsible for drug release.

In vitro swelling studies. In vitro swelling studies demonstrated that, Carbopol polymers showed very good water absorption characteristics, which was manifested by rapid increase of the diameter of the tablets i.e. swelling of the bilayered tablets. Initial diameter of the tablets was $13 \mathrm{~mm}$. After 6 hours, for Carbopol 934P the diameter of the tablets increased by $73 \%$ in distilled water, $50.4 \%$ in $0.1 \mathrm{~N} \mathrm{HCl}$ and $68 \%$ in phosphate buffer. For Carbopol 974P and 971P the diameter of the tablets increased by $76.3 \%$ and $78 \%$ in distilled water, $59 \%$ and $60.4 \%$ in $0.1 \mathrm{~N} \mathrm{HCl}$ and $64 \%$ and $70 \%$ in phosphate buffer 
respectively. Swelling was maximum for phosphate buffer. This indicates the ionization of the carboxylate groups of the polymers and repulsion between the native charges.

In vivo mucoadhesive performance of tablets without drug. Figure 2 shows the mucoadhesive performance of the tablets. All tablets adhered immediately to the gum and showed residence times above 6 hours. No tablets caused a bad taste, irritation or pain. All tablets proved to be well adhered to the gum and showed gel formation at the surface.

In vitro drug release study. In vitro drug release study was performed with Carbopol 934P NF. According to the water uptake rate and swelling study of three Carbopol polymers, it was noted that, Carbopol 971P NF showed the maximum swelling and fastest water uptake, which may cause inconvenience to the patients. On the other hand, Carbopol 974P NF provided a higher level of tack than Carbopol 971P NF or Carbopol 934P NF.

The bilayered tablets were prepared with Carbopol 934P NF alone at three different concentrations (80 mg, $140 \mathrm{mg}$ and $240 \mathrm{mg}$ ) and also in combination with HPMC at different concentrations to examine the effect on drug release. All tablets were submitted to in vitro release studies in sink conditions and monitored for 6 hours. Data were reported for only the period from 1 hour to 6 hours since the study was intended for buccal drug delivery.

Figure 3 shows drug release for tablets containing Carbopol 934P only (F1, F2 and F3). All tablets showed slow and gradual drug release throughout the experimental time. F3 tablets showed greater amount of drug release (51.238\%), whereas F1 tablets released only $30.92 \%$. The inclusion of higher percentages of $\mathrm{CP}$ provides prolonged release of drug through its properties of in situ gelling and slow dissolution. ${ }^{11}$

Figure 4 reports a comparative study of drug release for formulation F2 to F6. Drug release was gradual and the percentages were 45.33, 51.23, 44.46, 40.88 and $35.55 \%$ respectively. From figure 4, it is evident that as the concentration of HPMC increases and Carbopol content decreases in the formulations, drug release rate decreases. At $\mathrm{pH}$ 6.8, Carbopol 934P is present in the ionized state, so the polymer network gets loosened comparatively attributing for the faster drug release. ${ }^{12}$ Incorporating HPMC retarded drug release, evidenced from the drug release profiles of formulations F2 to F6.

Table 1. Fomrulation of bilayered tablets containing Lidocaine and Carbopol polymers

\begin{tabular}{|c|c|c|c|c|c|c|c|c|c|c|c|}
\hline \multirow[b]{3}{*}{$\mathbf{N}$} & \multicolumn{9}{|c|}{ Composition } & \multirow{2}{*}{\multicolumn{2}{|c|}{ Tableting time (s) }} \\
\hline & & \multicolumn{3}{|c|}{ First layer (mg) } & \multirow[b]{2}{*}{$\begin{array}{c}\text { Iron Oxide } \\
\text { Red }\end{array}$} & \multicolumn{4}{|c|}{ Second layer (mg) } & & \\
\hline & $\begin{array}{l}\text { Kollidon } \\
\text { SR }\end{array}$ & Talc & Aerosil & $\begin{array}{c}\text { Mg- } \\
\text { stearate }\end{array}$ & & Lidocaine & $\begin{array}{c}\text { Carbopol } \\
\text { 934P }\end{array}$ & $\begin{array}{c}\text { Carbopol } \\
974 \mathrm{P}\end{array}$ & $\begin{array}{c}\text { Carbopol } \\
\text { 971P }\end{array}$ & First & Second \\
\hline 1 & 150 & 3 & 4 & 3 & 2 & 50 & 240 & - & - & 5 & 60 \\
\hline 2 & 150 & 3 & 4 & 3 & 2 & 50 & - & 240 & - & 5 & 60 \\
\hline 3 & 150 & 3 & 4 & 3 & 2 & 50 & - & - & 240 & 5 & 60 \\
\hline
\end{tabular}

Table 2. Formulation of the bilayered buccal tablets with Carbopol 934P and HPMC

\begin{tabular}{|c|c|c|c|c|c|c|c|c|c|c|}
\hline \multicolumn{11}{|c|}{ Composition } \\
\hline \multirow[b]{2}{*}{$\mathrm{F}$} & \multicolumn{5}{|c|}{ First layer (mg) } & \multicolumn{3}{|c|}{ Second layer (mg) } & \multicolumn{2}{|c|}{ Tableting time (s) } \\
\hline & $\begin{array}{l}\text { Kollidon } \\
\text { SR }\end{array}$ & Talc & Aerosil & $\begin{array}{c}\text { Mg- } \\
\text { stearate }\end{array}$ & $\begin{array}{l}\text { Iron Oxide } \\
\text { Red }\end{array}$ & Lidocaine & $\begin{array}{c}\text { Carbopol } \\
\text { 934P }\end{array}$ & $\begin{array}{l}\text { HPMC } 15 \\
\text { cps }\end{array}$ & First & Second \\
\hline F1 & 150 & 3 & 4 & 3 & 2 & 50 & 240 & - & 5 & 60 \\
\hline $\mathrm{F} 2$ & 150 & 3 & 4 & 3 & 2 & 50 & 140 & - & 5 & 60 \\
\hline F3 & 150 & 3 & 4 & 3 & 2 & 50 & 80 & - & 5 & 60 \\
\hline $\mathrm{F} 4$ & 150 & 3 & 4 & 3 & 2 & 50 & 120 & 20 & 5 & 60 \\
\hline F5 & 150 & 3 & 4 & 3 & 2 & 50 & 100 & 40 & 5 & 60 \\
\hline F6 & 150 & 3 & 4 & 3 & 2 & 50 & 80 & 60 & 5 & 60 \\
\hline
\end{tabular}




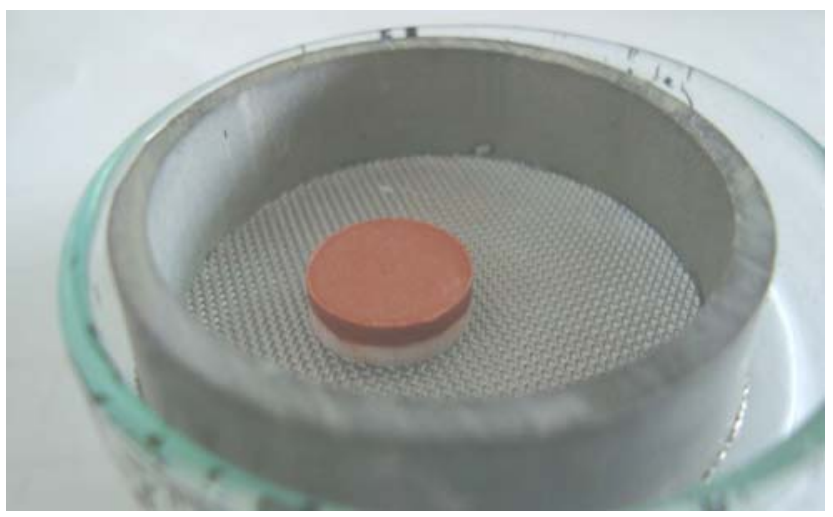

Figure 1. Device used to measure water uptake and swelling of the bilayered buccal tablets

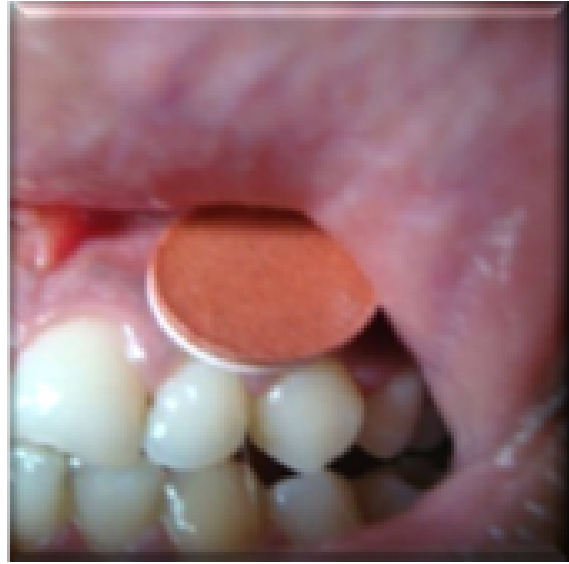

(a)

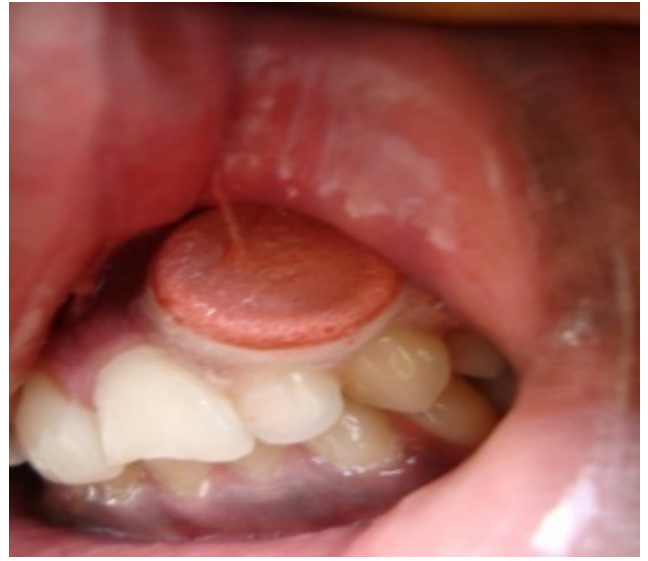

(b)

Figure 2: In vivo mucoadhesive performance of Carbopol Polymer, a) Immediately after application, (b) 6 hours after application.

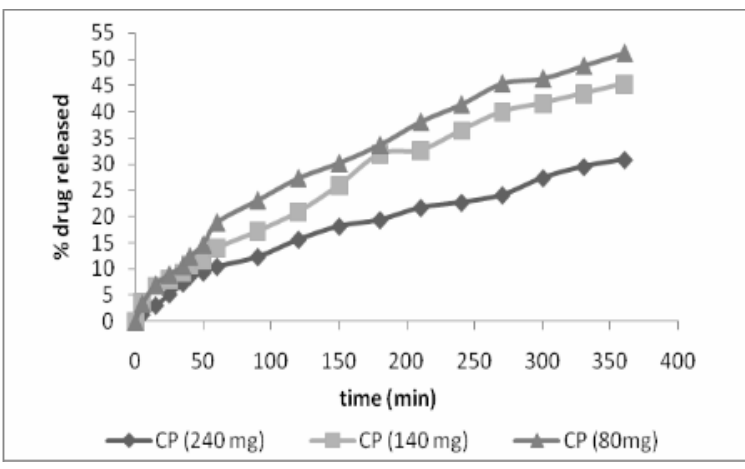

Figure 3. In vitro drug release study from Carbopol 934P at different concentration

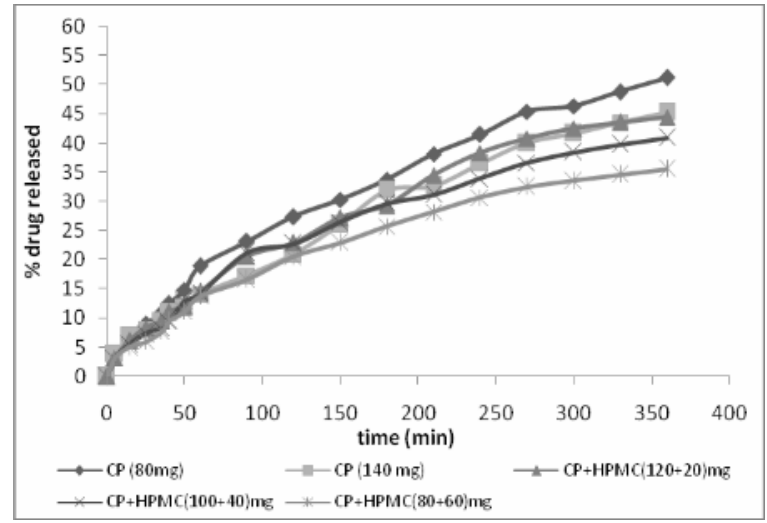

Figure 4. Comparison of In vitro drug release from Carbopol 934P and HPMC in combination 


\section{CONCLUSION}

Though HPMC is used in extended release dosage form, its mucoadhesive property is not satisfactory. The aim of this study started with a vision to observe the mucoadhesive properties of Carbopol polymers and their effectiveness to be used in buccal tablets. These studies showed that, Carbopol polymers absorbed water as well as gastrointestinal fluid many times of their weight and the diameter of the tablets also increased tremendously. In vivo mucoadhesive performance was also evaluated for these bilayered tablets. The tablets showed excellent mucoadhesive property for 6 hours. In vitro drug release was slower while using higher concentration of Carbopol polymer. In vitro release study in combination with HPMC showed that, with increasing concentration of HPMC, drug release was decreased. Because the results obtained by the newly developed methods are in excellent agreement with the reported findings, buccal adhesive tablet preparations with Carbopol polymers may be used as an ideal delivery system for local anesthetic agents.

\section{REFERENCES}

1. Harding, S.E., Davis, S.S. (BOB), Deacon, M.P. and Fiebrig, I. 1999. Biopolymer Mucoadhesives. Biotechnol. Genet. Eng. Rev. 16, 41-86

2. Park, H. and Robinson J.R. 1985. Physico-chemical properties of water insoluble polymers important to mucin/epithelial adhesion, J. Control. Rel. 2, 47-57
3. Nangia, A. 2006. Bioadhesives for targeted oral drug delivery, drug delivery report spring/summer, technology overviews. pp. 25-32

4. Krishna, S.S., Ray, S. and Thakur, R.S. 2006. Formulation and evaluation of mucoadhesive dosage form containing rosiglitazone maleate. Pak. J. Pharm. Sci. 19, 208-213

5. Peppas, N.A. and Buri, N.A. 1985. Surface,Interfacial and molecular aspects of polymer bioadhesion on soft tissue, $J$. Control Rel. 2, 257-275

6. Shojaei, A.H., Zhou, S. and Li, X. 1998. Transbuccal delivery of acyclovir (III): Feasibility, system design and in vitro permeation studies, J. Pharm. Pharmaceut 1, 66-73

7. Buccal Delivery of Drugs, PCT Inc. Pharmaceutical applications, www.pctincusa.com, Company Overview, p 1.

8. Bulletin 2. 2002. Product and Regulatory Guide, Noveon Company Technical Literature, p. 2.

9. Hosmani, A.H.. 2006. Carbopol and its Pharmaceutical Significance: A Review, Pharmainfo.net, 4, 5

10. Perioli, L., Ambrogi, V., Giovagnoli, S., Ricci, M., Blasi, M. and Rossi, C., 2007, Mucoadhesive bilayered tablets for buccal sustained release of flurbiprofen, AAPS Pharm. Sci. Tech. 8, Article 54, E1-E8

11. Patel, V.M., Prajapati, B.G. and Patel, M.M. 2007. Formulation, evaluation and comparison of bilayered and multilayered mucoadhesive buccal devices of propanold hydrochloride, AAPS Pharm. Sci. Tech. 8, Article 22, E147E154

12. Patel, J., Patel, R., Amin A. and Patel, M. 2005. Buccal mucoadhesive Patches of lignocaine hydrochloride: an alternative for infiltration anesthesia for tooth extraction, Drug Deliv. Technol. 5. 